\title{
Gaussian Process for Interpreting Pulsed Eddy Current Signals for Ferromagnetic Pipe Profiling
}

\author{
Nalika Ulapane*, Alen Alempijevic*, Teresa Vidal-Calleja*, Jaime Valls Miro*, Jeremy Rudd ${ }^{\dagger}$, Martin Roubal ${ }^{\dagger}$ \\ ${ }^{*}$ Centre for Autonomous Systems, University of Technology Sydney, Australia \\ $\dagger$ Rock Solid Group, Melbourne, Australia
}

\begin{abstract}
This paper describes a Gaussian Process based machine learning technique to estimate the remaining volume of cast iron in ageing water pipes. The method utilizes time domain signals produced by a commercially available pulsed Eddy current sensor. Data produced by the sensor are used to train a Gaussian Process model and perform inference of the remaining metal volume. The Gaussian Process model was learned using sensor data obtained from cast iron calibration plates of various thicknesses. Results produced by the Gaussian Process model were validated against the remaining wall thickness acquired using a high resolution laser scanner after the pipes were sandblasted to remove corrosion. The evaluation shows agreement between model outputs and ground truth. The paper concludes by discussing the implications or results and how the proposed method can potentially advance the current technological setup by facilitating real time pipe profiling.

Keywords-ferromagnetic, Gaussian process, machine learning, non-destructive testing, pulsed Eddy current, sensor model
\end{abstract}

\section{INTRODUCTION}

Exposure of ferromagnetic water mains to aggressive environmental conditions and deleterious reactions can lead to significant deterioration so as to undermine their ability to function desirably and eventually fail through bursts [1]. Therefore, the prediction of a pipe's remaining life, especially for critical water mains, is important for developing effective renewal programs to manage pipe infrastructure and reducing the incidence of catastrophic failures. Better understanding of the current condition and performance of buried water mains and sewer pressure mains is an important first step to help achieve improved understanding of remaining life. This is where the requirement for improved nondestructive metal pipe condition assessment techniques arise.

Techniques such as Pulsed Eddy Currents (PEC), Remote Field Eddy Currents, Magnetic Flux Leakage (MFL) and Ultrasounds are commonly used for the purpose of examining defects of ferromagnetic pipes [2]. Specifically, PEC technology is being used for evaluating thickness of conducting material test pieces [3], [4], [5], corrosion detection [6], surface and subsurface defect detection [7] and pipe inspection [1], [6]. PEC technology usually characterises and quantifies defects, sensor liftoffs and material thicknesses by means of evaluating features extracted from the sensor response. Among the many possible features, the difference curve features [4], [7], [8], logarithmic response features [9], [5], frequency spectrum features [10], unique characteristics of sensor response [7], principal components [11] and integral features [3] are the ones predominantly used. Certain features extracted exhibit straightforward relationships to properties of the test piece when the other properties are controlled. However, the problem is not that straight when the technology is used on cast iron pipes since there is minimal prior knowledge about the true nature of the test pieces and they are subjected to more complex contamination in the form of graphitisation and surface and subsurface defects of arbitrary shapes and sizes.

Prior work on applying machine learning principles for Eddy current profiling [12], [13] has not addressed the sophisticated problem of profiling the test piece where both surface and subsurface defects of arbitrary shapes are present. For instance [12] classifies cracks based on the straight crack hypothesis and the proposed method is limited when trying to address arbitrary shaped cracks. However, as mentioned in [12], more general problems may be solved by increasing the evaluated parameters in the training data set. The form of defects present in aged cast iron pipes is not limited. Thus, existing feature extraction techniques are not transferable to profiling cast iron pipes with complex geometry, the full sensor response instead of few features for training could be utilised. As cast iron material is ferromagnetic, this results in weak penetration of Eddy currents because of the skin effect and further exacerbates the need to exploit the full response that can be obtained. All work related to PEC signal interpretation found in literature, even the ones using machine learning [12], [13], have been attempted on nonferromagnetic materials. However, [14] is one exception where PEC technology has been used in conjunction with Remote Field Eddy Currents for testing ferromagnetic tubes.

This paper brings novelty in using the Bayesian estimation framework incorporating Gaussian processes (GPs) [15] to estimate the remaining volume of cast iron. GPs are nonparametric models that can be used to solve non-linear regression problems such as the one in hand. GPs have been recently applied in this context to identify defects in steel plates with MLF sensors [16]. In this paper, PEC sensor signals are used as input for this non-parametric model. The GP model is trained using cast iron calibration plates of various thicknesses. Thereafter the trained model is used to estimate the remaining wall thickness of in-situ pipes. Validation of the estimated results is performed on the pipes once they are sandblasted to remove corrosion. The evaluation is performed on two $1 \mathrm{~m}$ sections of a cast iron pipe with $600 \mathrm{~mm}$ external diameter showing agreement between model outputs and ground truth with 3.08 and 
$1.71 \mathrm{~mm}$ root mean square error and standard deviation respectively.

The paper unfolds under four sections from here onwards. First the theory behind the sensor architecture and the GPs approach are explained in section II. It is followed by section III which explains the experimental work carried out along with the procedures of training, testing and validating the GP model. Section IV includes graphical and numerical results which exhibit the validity of the GPs outputs. Finally, the concluding remarks are included in section $\mathrm{V}$ along with the implication of results and potential improvements to the proposed method.

\section{APPROACH}

The proposed approach uses supervised learning to train a non-parametric model, which gets input features extracted from the PEC sensor signals and produces an estimate of the remaining wall thickness of the ferromagnetic material underneath the sensor. As can be appreciated from the aforementioned, the ability to estimate remaining wall thickness comprises of three primary components. Firstly, a sensor architecture capable of energising and measuring the Eddy currents in a ferromagnetic material, following a subsampling (feature extraction) step and a machine learning framework, all of which are detailed in the following sub-sections.

\section{A. Sensor principles}

In a typical PEC sensor, the primary coil is excited with a voltage pulse. This pulse creates a time varying magnetic field around the sensor, the resulting magnetic field induces Eddy currents in the conducting test piece (i.e. cast iron pipe) as shown in Fig. 1. Induced Eddy currents create a magnetic field, which opposes the field generated by the exciter coil. The resultant time varying magnetic field induces a voltage in the detection coil. This induced voltage is the output signal produced by the sensor and it takes the shape of a decay curve as shown in Fig. 2. The time dependant decay curves uniquely characterise properties of the test piece. The output signal (induced voltage) produced by the antenna are filtered, digitally processed and recorded. Fig. 3 shows a basic block diagram of the macro view of the full sensor setup. However, details about the dimensions of the antenna, amplitude and frequency of the sensor excitation sequence and the internal electronics are omitted. The approach presented herewith is generic in nature and does not critically depend on these parameters.

The fundamental principle of the sensor, as described in [17], is as follows. If the resultant magnetic vector potential at an arbitrary location inside the domain of the detection coil is expressed as $A$, the coil current density $J$ at that location is given by

$$
\nabla^{2} A-\mu \sigma \frac{d A}{d t}=-\mu J
$$

where $\mu$ and $\sigma$ are the permeability and conductivity of the detection coil material.

When the current density at a point is known, the electric field intensity $E$ at that point is given by $J=\sigma E$ and

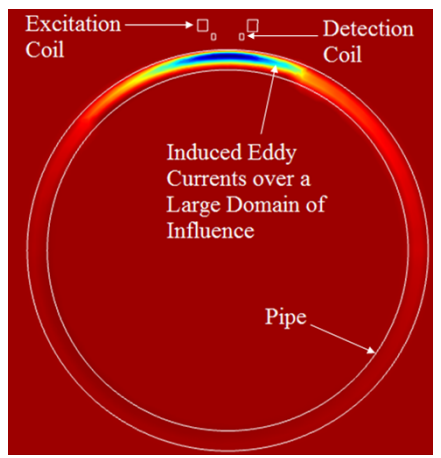

Fig. 1. Cross sectional view of the Comsol Multiphysics ${ }^{\circledR}$ model showing the Eddy current induction phenomenon.

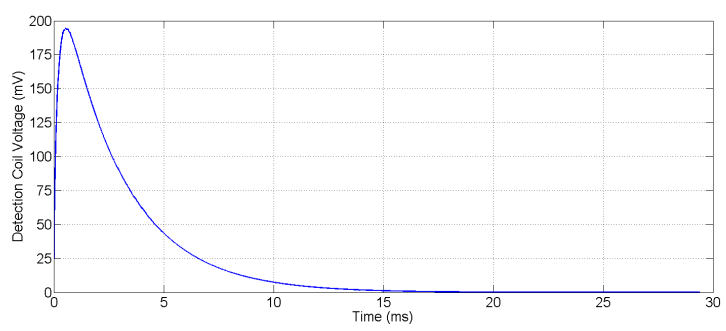

Fig. 2. Induced voltage of the detection coil known as decay curve.

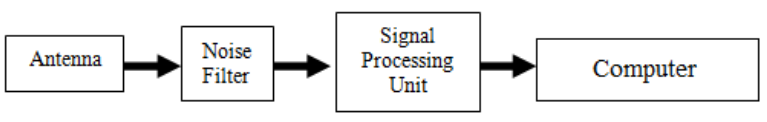

Fig. 3. Diagram of the full sensor setup.

therefore, it is known that the detection voltage $V$ can be calculated by performing the line integral

$$
V=\int_{C} E d l
$$

along the total length of the detection coil.

To model any type of Eddy current sensor requires solving (1) and (2) and this work is no exception. Thus, the sensor response shown in Fig. 2 was generated by solving (1) and (2). To illustrate the anticipated sensor response, a solution is provided using Finite Element Analysis (FEA) [17] with the aid of the commercial FEA package Comsol Multiphysics ${ }^{\circledR}$. Fig. 4 shows the complete 3D model of the PEC sensor placed above the cast iron pipe, made in the FEA package. The phenomenon of Eddy current induction in the pipe can be visualized in the cross sectional image in Fig. 1. It is clear from Fig. 1 that the domain of influence (the area in which the Eddy currents are induced) is much larger than the area physically covered by the sensor.

\section{B. Feature Extraction}

Feature extraction is a well-known mechanism to reduce the dimensionality of the data. It maps raw sensor signals into a lower dimensional space. Features, in this case, are derived from full time-varying voltage signals produced by 


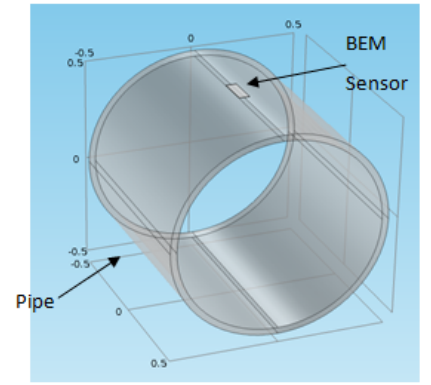

Fig. 4. FEA simulation model of the sensor placed on a cast iron pipe, made in Comsol Multiphysics ${ }^{\circledR}$

the sensor. The voltage decay curve obtained by the sensor, shown in Fig. 2 is subsampled with a logarithmic timescale similar to that in [18]. Thus, a subsampled version of the decay curve is produced with a very high sampling rate closer to the beginning of the decaying section of the curve at $1 \mathrm{~ms}$ and the last sampled instance being equal at the total signal duration of $58 \mathrm{~ms}$. A total of 24 subsamples were acquired in this interval as illustrated in Fig. 5 and used as features in the subsequent machine learning approach.

\section{Gaussian Process Formulation}

Estimating thickness from PEC sensor signals can be formulated as a non-linear regression problem. Gaussian Process models are a powerful tool to solve such regression problems. GPs can be thought of as a Gaussian prior over the function space mapping inputs $x$ and outputs $f(x)$. It is completely specified by its mean function $\mu=E[f(x)]$ and the covariance function $\Sigma=E\left[(f(x)-\mu)\left(f(x)^{\top}-\mu^{\top}\right)\right][15]$.

Let $[X, Y]$ be the training data set drawn by the noisy process $y_{i}=f\left(x_{i}\right)+\epsilon$, where $X=\left[x_{1}, x_{2}, \ldots, x_{m}\right]^{T}$ be a matrix of training inputs that in our particular case corresponds to the features extracted from the PEC signal and $Y=\left[y_{1}, y_{2}, \ldots, y_{m}\right]^{T}$ be a vector of training labels, which are the corresponding cast-iron plates thickness. A Gaussian Process estimates posterior distributions over functions $f$ from the training data $[X, Y]$. Although the functions are infinitely dimensional, the GP model is used to infer, or

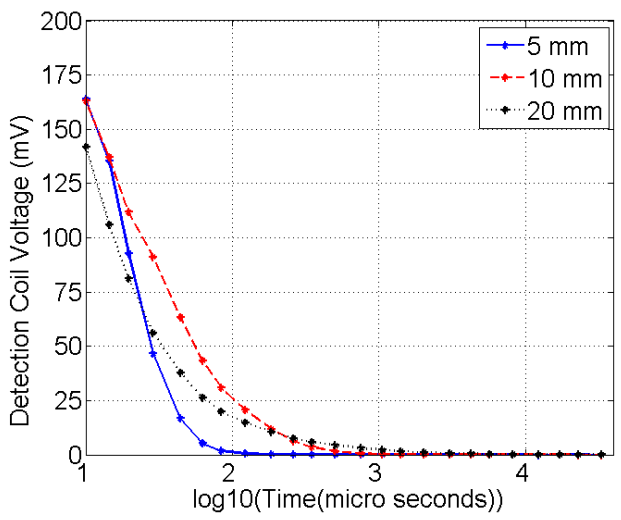

Fig. 5. Subsampled logarithmic timescale decay curves. predict, function values at a finite testing set of prediction points $X^{*}=\left[x_{1}^{*}, x_{2}^{*}, \ldots, x_{n}^{*}\right]^{T}$.

To apply a GP framework to this regression problem, one must first select a kernel $K(X, X)$ whose elements are given by $k_{i, j}=k\left(x_{i}, x_{j}\right)$. This specifies the kind of functions that are expected, before any data have been seen. Technically, the kernel places a prior likelihood on all possible functions. After evaluating a number of commonly used kernels, the squared exponential kernel has been chosen for this work. It is defined as

$$
k\left(x_{i}, x_{j}\right)=\alpha^{2} \exp \left\{-\frac{1}{2 \beta^{2}}\left(x_{i}-x_{j}\right)^{2}\right\},
$$

where $\alpha$ and $\beta$ represent its hyper-parameters and together with sensor noise variance $\sigma_{n}$ are learned from the training data. The GP model is trained by minimizing the negative $\log$ marginal likelihood in (4) with respect to $\theta=\left\{\alpha, \beta, \sigma_{n}\right\}$.

$$
-\log p(Y \mid X, \theta)=\frac{1}{2} Y^{T} \Sigma^{-1} Y+\frac{1}{2} \log |\Sigma|+\frac{m}{2} \log (2 \pi),
$$

where the covariance function is given by

$$
\Sigma=K(X, X)+\sigma_{n}^{2} I .
$$

The combination of the training data and the kernel induces not only the most likely state, but also a full posterior probability distribution. The basic GP regression equations are given by

$$
\begin{aligned}
\mu^{*}= & K\left(X^{*}, X\right)\left\{K(X, X)+\sigma_{n}^{2} I\right\}^{-1} y \\
\Sigma^{*}= & K\left(X^{*}, X^{*}\right)+\sigma_{n}^{2} I-K\left(X^{*}, X\right) \\
& \left\{K(X, X)+\sigma_{n}^{2} I\right\}^{-1} K\left(X, X^{*}\right),
\end{aligned}
$$

where $I$ is the corresponding identity matrix.

The expected pipe wall thickness for the testing input vector $X^{*}$ will be therefore given by the mean of the posterior distribution $\mu^{*}$ and the associated uncertainty will be given by the covariance $\Sigma^{*}$.

\section{Methodology}

The complete proposed methodology is presented in Fig. 6. Three main tasks were carried out in this work: training the GP model, generating the pipe profiles and validating the model outputs. The methods to accomplish these tasks are explained in detail in the following subsections.

\section{A. Training the GP Model}

Training data were obtained by scanning cast iron calibration blocks of thicknesses 1 through 10 and then $12,14,16,18,20,22,25,30$ and $35 \mathrm{~mm}$, as generally done by the PEC technology provider. Note that the more data used for training the less uncertainty associated to the output of the GP model. The training data for this work was obtained by maintaining the sensor liftoff constant at $0 \mathrm{~mm}$. This replicates the physical gap that will exist between the sensor and the pipe when obtaining readings in-situ as in Fig. 7. 


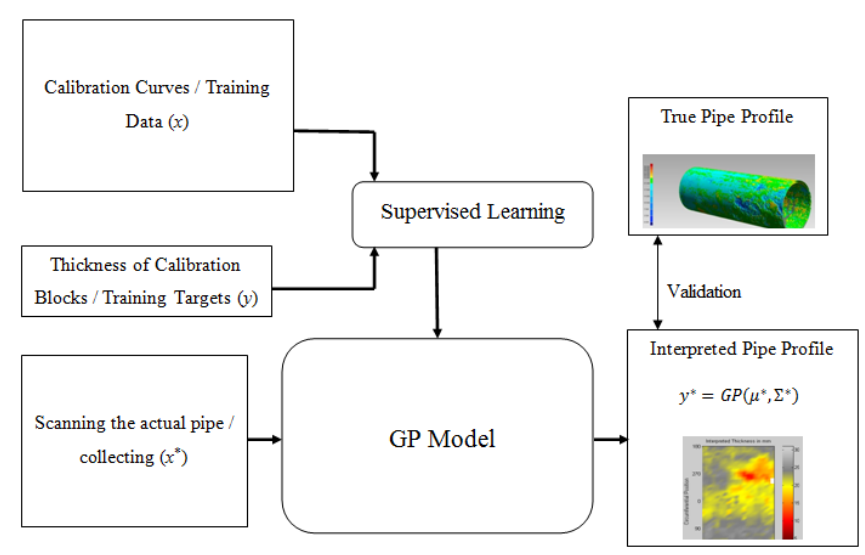

Fig. 6. Complete methodology of training the GP model, generating pipe profiles and validating.

As mentioned previously in section II-B, the subsampled logarithmic time domain decay curves were used as feature vectors for this work to overcome the restrictions associated with standard features extracted from the curves. The set of subsampled decay curves together with the associated thickness were used as to learn the GP model.

An example of the posterior drawn by the GP inference for two selected features is shown in Fig. 8. The non-linear functions associated with these features and captured by the GP model show complimentary discriminative powers. While feature 10 discriminates well in the region between 8-16 mm thickness as illustrated in Fig. 8(a), feature 18 holds discriminative power in the $14-25 \mathrm{~mm}$ region as per Fig. 8(b). Additionally, higher uncertainty in the GP model is observed in regions where insufficient training data is provided. For example, an inference of $13 \mathrm{~mm}$ thickness has demonstrably high uncertainty, whereas inferences of $12 \mathrm{~mm}$ and $14 \mathrm{~mm}$ where training data is provided have substantially lower uncertainty bounds as denoted in Fig. 8(a). Finally, it can also be ascertained that neither feature in Fig. 8 has substantial discriminative power in thickness above $25 \mathrm{~mm}$. This is caused by a combination of insufficient training data and reduced eddy currents being generated further away from the exciter, both factors will be addressed in future work.

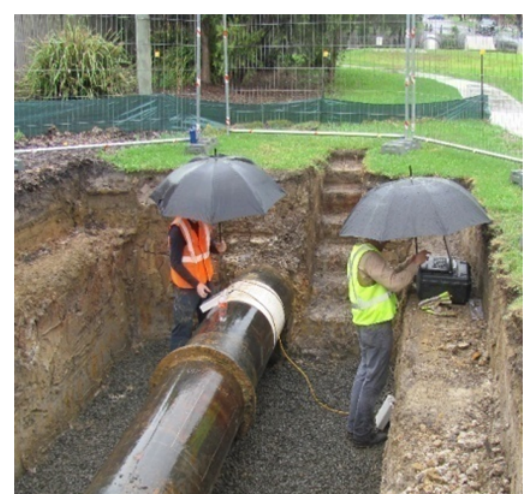

Fig. 7. Obtaining testing data (BEM scanning being done on the test bed).

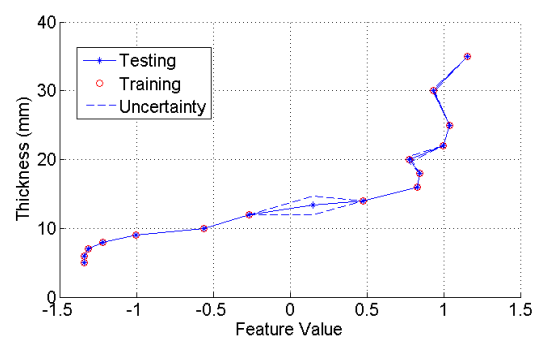

(a) Feature 10

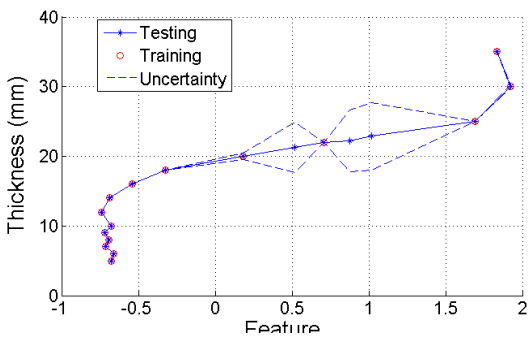

(b) Feature 18

Fig. 8. GP inference of thickness for two features from the considered feature vector

\section{B. Obtaining Testing Data}

Testing data were obtained by scanning the full circumference of pipe segments of $1 \mathrm{~m}$ length along the pipe axis on the test bed allocated for this work. Reference [2] provides details about the $1.5 \mathrm{~km}$ test bed, two $1 \mathrm{~m}$ pipe segments were identified by Sydney Water as possibly defective and required more in depth inspection. Fig. 7 shows how BEM scanning is done on the pipe test bed. To account for variability in electro-magnetic properties of cast iron the training data and testing data from each pipe segment are jointly normalised and standardised. As estimation of thickness is not performed in real-time, this limitation will be addressed in future work.

\section{Generating 2D Pipe Profiles}

Once the GP model is trained, the testing decay curves obtained from the test bed are used to infer the pipe profiles as $2 \mathrm{D}$ thickness plots along with the uncertainty. Features are extracted as described in section II-B and, (6) and (7) are used to produce the estimated thickness.

\section{Validating the GP Model Outputs}

The test bed pipe segments were exhumed and both surfaces (external and internal) were sandblasted to strip off any rust or graphitization in order to obtain segments with clean metal [2]. The external and internal surfaces of the sandblasted pipe segments were scanned using a 3D laser scanner and accurate 3D pipe profiles were generated using a ray tracing algorithm described in [2]. Shown in Fig. 9 is an example of the obtained 3D laser profile of a scanned pipe segment.

Note that the resolution of the laser profiles is $2 \mathrm{~mm}$, while that of the PEC sensor is $5 \mathrm{~cm}$. Therefore, the 3D profile thicknesses were averaged to obtain the 2D thickness plots for qualitative and quantitative comparison. The GP 
inference results were compared with the ground truths to evaluate the accuracy as presented in the following section.

\section{RESUlTS AND DISCUSSION}

This section includes the results produced by the GP model and the comparison against the ground truth. As mentioned above results of two pipe segments are included.

Fig. 10 and 11 show the GP inferred thickness plots and corresponding uncertainties of the two segments. The thickness and uncertainty plots represent the profile of a $660 \mathrm{~mm}$ diameter full circumferential pipe segment of 1000 $\mathrm{mm}$ in length along the $x$ direction which is parallel to the axis of the pipe. A comparison of the inferred profiles against the ground truth and the profiles produced by the technology provider (RSG) are shown in Fig. 12 and 13. The white sections in the GP plots are sensor readings that were ignored due to anomalies caused by not having the hand held BEM sensor stable for a sufficient duration when acquiring data for that section of the pipe.

A quantitative comparison is presented in Table I and II showing that the results produced by the proposed GP approach are marginally better (closer to the ground truth) than the results produced by RSG. The results show that for both segments, the RSG and our GP inference, are in accordance with the ground truth. The proposed approach, however, produces uncertainty plots that give a measure of the reliability on the estimated thickness as shown on the right hand side of Figs. 10 and 11. The uncertainty varies depending on how close the estimated thickness is to the training data, the more training samples the more accurate results. Fig. 14 highlights a region where the uncertainty is

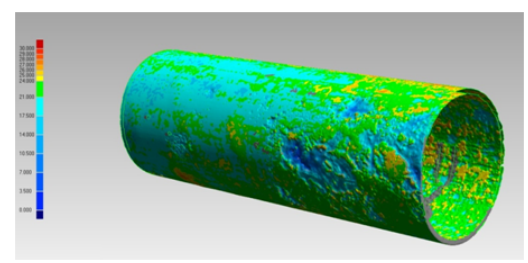

Fig. 9. 3D laser profile of the scanned pipe (Ground Truth).
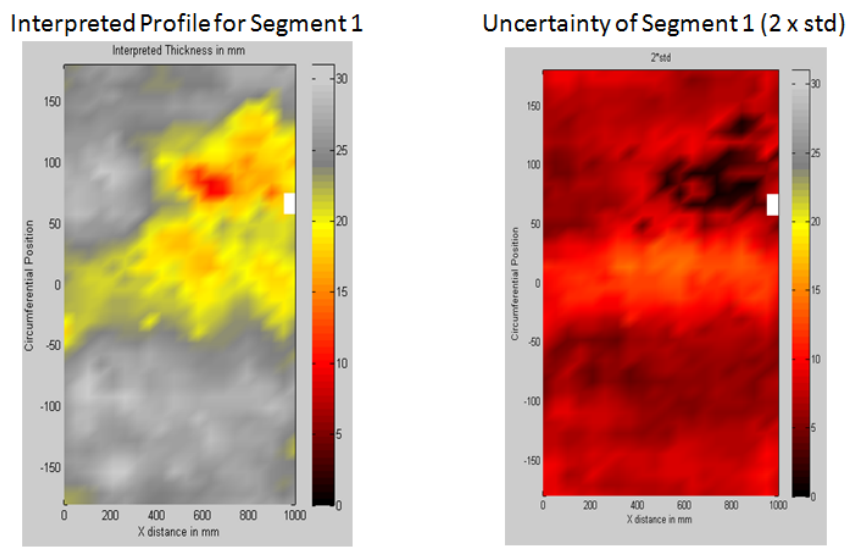

Fig. 10. Interpreted pipe profile and uncertainty of segment 1 .
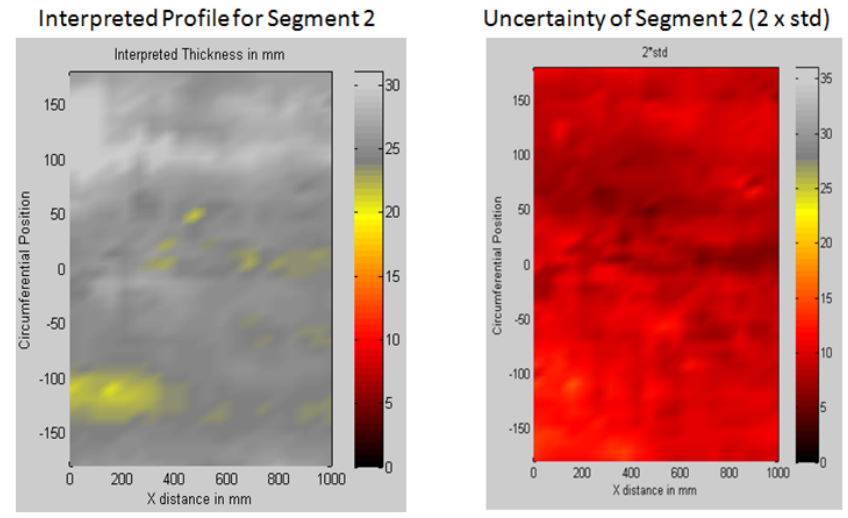

Fig. 11. Interpreted pipe profile and uncertainty of segment 2.

TABLE I

COMPARISON STATISTICS OF SEGMENT 1, THE ERROR BETWEEN INTERPRETED THICKNESS AND GROUND TRUTH

\begin{tabular}{lll}
\hline Value & GP & RSG \\
\hline Mean & $2.71 \mathrm{~mm}$ & $2.96 \mathrm{~mm}$ \\
Standard Deviation & $1.76 \mathrm{~mm}$ & $1.50 \mathrm{~mm}$ \\
Root Mean Square & $3.23 \mathrm{~mm}$ & $3.29 \mathrm{~mm}$ \\
Minimum & $0.02 \mathrm{~mm}$ & $0 \mathrm{~mm}$ \\
Maximum & $10.4 \mathrm{~mm}$ & $7.28 \mathrm{~mm}$ \\
\hline
\end{tabular}

low when estimating areas with lower thicknesses, implying higher reliability. The underlying reason is the larger amount of training data available for lower thicknesses.

\section{CONCLUSIONS}

This paper proposed a GP based machine learning technique for interpreting PEC signals for profiling the remaining wall thickness in ferromagnetic cast iron water pipes. The approach utilises the full sensor response, subsampling the original signal on a logarithmic scale to produce a feature vector which is different from the specific feature based work available in literature. The Gaussian Process model
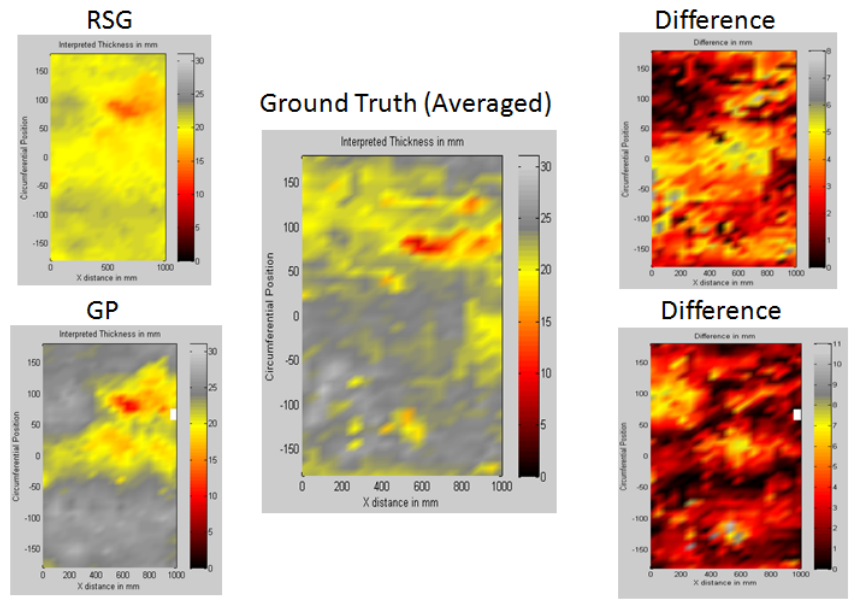

Fig. 12. Pipe segment 1(Comparison against Ground Truth). 


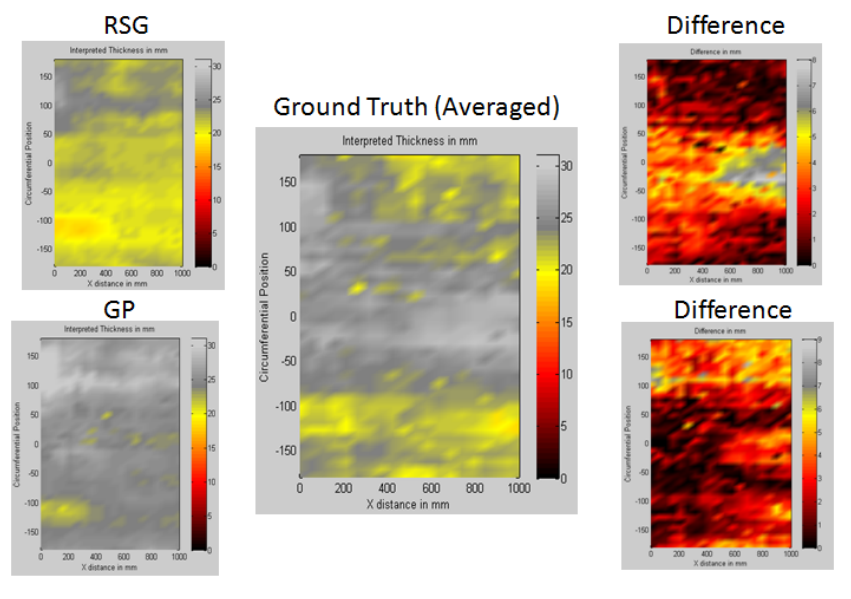

Fig. 13. Pipe segment 2(Comparison against Ground Truth).

TABLE II

COMPARISON STATISTICS OF SEGMENT 2, THE ERROR BETWEEN INTERPRETED THICKNESS AND GROUND TRUTH

\begin{tabular}{lll}
\hline Value & GP & RSG \\
\hline Mean & $2.41 \mathrm{~mm}$ & $2.48 \mathrm{~mm}$ \\
Standard Deviation & $1.67 \mathrm{~mm}$ & $1.76 \mathrm{~mm}$ \\
Root Mean Square & $2.93 \mathrm{~mm}$ & $3.03 \mathrm{~mm}$ \\
Minimum error & $0 \mathrm{~mm}$ & $0 \mathrm{~mm}$ \\
Maximum error & $8.14 \mathrm{~mm}$ & $7.90 \mathrm{~mm}$ \\
\hline
\end{tabular}
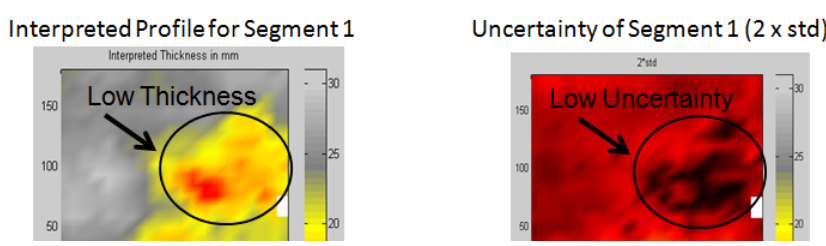

Fig. 14. Zoomed view of Pipe segment 1 and associated Uncertainty.

was trained using sensor signals obtained from cast iron calibration plates of various thicknesses. Results produced by the Gaussian Process model were validated against the remaining wall thickness with agreement observed between model outputs and ground truth (root mean square error 3.08 and standard deviation $1.71 \mathrm{~mm}$ ). Profiling in this work is coarse due to the constraints and design of the sensor used.

Future work considers fusing several PEC sensor measurements in overlapping configurations to produce higher resolution pipe profiles and altering the excitation waveform to enable discrimination of larger wall thicknesses. There is also room for designing more sophisticated PEC sensor architectures to overcome obstacles like skin effect for accurate profiling of ferromagnetic materials.

\section{ACKNOWLEDGMENT}

This publication is an outcome from the Critical Pipes Project funded by Sydney Water Corporation, Water Research Foundation of the USA, Melbourne Water, Water Corporation (WA), UK Water Industry Research Ltd, South Australia Water Corporation, South East Water, Hunter Water
Corporation, City West Water, Monash University, University of Technology Sydney and University of Newcastle. The research partners are Monash University (lead), University of Technology Sydney and University of Newcastle.

\section{REFERENCES}

[1] B. Rajani and Y. Kleiner, "Non-destructive inspection techniques to determine structural distress indicators in water mains," Evaluation and Control of Water Loss in Urban Water Networks, pp. 21-25, 2004.

[2] J. V. Miro, J. Rajalingam, T. Vidal-Calleja, F. de Bruijn, R. Wood, D. Vitanage, N. Ulapane, B. Wijerathna, and D. Su, "A live test-bed for the advancement of condition assessment and failure prediction research on critical pipes," in Proceedings of the Leading-Edge Strategic Asset Management Conference (LESAM13), 2013.

[3] D. Chen, Q. Ji, H. Zhang, and L. Zhao, "Application of pulsed eddy current in plate thickness evaluation," in 4th IEEE Conference on Industrial Electronics and Applications (ICIEA), 2009, pp. 3286-3288.

[4] C. Tai, J. H. Rose, and J. C. Moulder, "Thickness and conductivity of metallic layers from pulsed eddy current measurements," Review of Scientific Instruments, vol. 67, no. 11, pp. 3965-3972, 1996.

[5] V. De Haan and P. de Jong, "Simultaneous measurement of material properties and thickness of carbon steel plates using pulsed eddy currents," in 16th Word Conference on Non-Destructive Testing in Montreal, 2004.

[6] H. W. Liu, S. P. Zhan, Y. H. Du, and P. Zhang, "Study on pulsed eddy current nondestructive testing technology for pipeline corrosion defects based on finite element method," Applied Mechanics and Materials, vol. 120, pp. 36-41, 2012.

[7] G. Y. Tian and A. Sophian, "Defect classification using a new feature for pulsed eddy current sensors," NDT \& E International, vol. 38, no. 1, pp. 77-82, 2005.

[8] Y. He, F. Luo, M. Pan, F. Weng, X. Hu, J. Gao, and B. Liu, "Pulsed eddy current technique for defect detection in aircraft riveted structures," NDT \& E International, vol. 43, no. 2, pp. 176-181, 2010.

[9] C. Nelson, C. Cooperman, W. Schneider, D. Wenstrand, and D. Smith, "Wide bandwidth time-domain electromagnetic sensor for metal target classification," IEEE Transactions on Geoscience and Remote Sensing, vol. 39, no. 6, pp. 1129-1138, 2001.

[10] J. García-Martín, J. Gómez-Gil, and E. Vázquez-Sánchez, "Nondestructive techniques based on eddy current testing," Sensors, vol. 11, no. 3, pp. 2525-2565, 2011.

[11] A. Sophian, G. Y. Tian, D. Taylor, and J. Rudlin, "A feature extraction technique based on principal component analysis for pulsed eddy current ndt," NDT \& E International, vol. 36, no. 1, pp. 37-41, 2003.

[12] A. Bernieri, L. Ferrigno, M. Laracca, and M. Molinara, "Crack shape reconstruction in eddy current testing using machine learning systems for regression," IEEE Transactions on Instrumentation and Measurement, vol. 57, no. 9, pp. 1958-1968, 2008.

[13] Y. He, M. Pan, F. Luo, D. Chen, and X. Hu, "Support vector machine and optimised feature extraction in integrated eddy current instrument," Measurement, 2012.

[14] D. Vasic, V. Bilas, and D. Ambrus, "Pulsed eddy-current nondestructive testing of ferromagnetic tubes," IEEE Transactions on Instrumentation and Measurement, vol. 53, no. 4, pp. 1289-1294, 2004.

[15] C. E. Rasmussen, Gaussian processes for machine learning. MIT Press, 2006.

[16] B. Wijerathna, T. Vidal-Calleja, S. Kodagoda, Q. Zhang, and J. Valls Miro, "Multiple defect interpretation based on gaussian processes for mfl technology," Proc. SPIE Nondestructive, vol. 8694, pp. 86941Z-86941Z-12, 2013. [Online]. Available: http://dx.doi.org/10.1117/12.2009966

[17] R. Ludwig and X.-W. Dai, "Numerical and analytical modeling of pulsed eddy currents in a conducting half-space," IEEE Transactions on Magnetics, vol. 26, no. 1, pp. 299-307, 1990.

[18] D. R. Desjardins, G. Vallires, P. P. Whalen, and T. W. Krause, "Advances in transient (pulsed) eddy current for inspection of multilayer aluminum structures in the presence of ferrous fasteners," AIP Conference Proceedings, vol. 1430, no. 1, pp. 400-407, 2012. 